\title{
Modeling the bistable transition between cell phenotypes during limb
} precartilage condensation

\author{
T. Glimm ${ }^{1}$ B. Kaźmierczak ${ }^{2}$, C. Cui ${ }^{3}$, S.A. Newman ${ }^{4}$ and R. Bhat ${ }^{5}$ \\ ${ }^{1}$ Western Washington University, Bellingham, WA, USA; ${ }^{2}$ Institute of Fundamental \\ Technological Research Polish Academy of Sciences, Warsaw, Poland; ${ }^{3}$ Janssen Research and \\ Development, LLC, Raritan, NJ, USA; ${ }^{4}$ New York Medical College, New York, NY, USA; \\ ${ }^{5}$ Molecular Reproduction, Development and Genetics, Indian Institute of Science, Bangalore \\ 50012, India
}

\begin{abstract}
Previous work showed that Gal-8 and Gal-1A, two proteins belonging to the galactoside-binding galectin family, are the earliest determinants of the patterning of the skeletal elements of embryonic chicken limbs, and further, that their experimentally determined interactions in the embryonic limb bud can be interpreted through a reaction-diffusion-adhesion framework. Here, we use an ordinary differential equation-based approach to analyze the intrinsic switching modality of the galectin reaction network and characterize the states of the network independent of the diffusive and adhesive arms of the patterning mechanism. We identify two steady states: where the concentrations of both the galectins are respectively, negligible, and very high. We provide an explicit Lyapunov function, which shows that there are no periodic solutions. In an extension of the model with sigmoidal galectin production terms, we show that an analogous bistable switch-like system arises via saddle-node bifurcation from a monostable one. Our model therefore predicts that the galectin network may exist in low expression and high expression states separated in space or time without any intermediate states. We verify these predictions in experiments performed with high density micromass cultures of chick limb mesenchymal cells and observe that cells inside and outside the precartilage protocondensations exhibit distinct behaviors with respect to galectin expression, motility, and spreading behavior on their substratum. The interactional complexity of the Gal-1 and -8-based patterning network is therefore sufficient to partition the mesenchymal cell population into two discrete cell types, which can be spatially patterned when incorporated into an adhesion and diffusion-enabled system. Keywords: limb development, galectins, cell state transition, switch-like regulatory network, ordinary differential equations, Lyapunov function, saddle-node bifurcation
\end{abstract}

\section{Introduction}

Cell differentiation during embryogenesis has long been recognized to have features in common with multistable systems of nonlinear dynamics and discrete Boolean networks. This resemblance has provided motivation for mathematical and computational models of development over the past half-century (Kauffman (1969); Thom (1976); Keller (1994); Furusawa and Kaneko (2012); Huang (2012)). The analogy is appealing for several reasons: an organism's genes (collectively its genome) and the RNA and protein molecules they specify can be conceived as a highly connected auto- and cross-regulatory multi-component system. Although the number of constituents 
(including alternative splice forms and covalently modified proteins) range in thousands in animals, for example, the number of distinct cell types are in the tens to hundreds (more than 250 in humans), suggesting that cell types may represent the discrete stationary states (time-independent solutions) or attractors of dynamical systems of gene regulatory networks (GRNs). Further, cell types are not only well-separated in the multidimensional state space defined by concentrations of gene products or transcription factors, they are also robust under perturbation, i.e., they exhibit stability in the face of physiological noise and transition between states along well-defined pathways during ontogeny or under experimental manipulation (Steinacher et al. (2016); Goode et al. (2016); Srivastava (2006)). All of this strengthens the analogy between cell types and the attractors of complex systems.

Analogies of developmental cell type transitions to dynamical systems also have severe limitations, however. The compositional differences (i.e., location in state space) between attractor states of a multicomponent dynamical system are a purely mathematical effect. Cell types, in contrast, embody coherent functions within their respective tissues: contractile muscles, supportive bones, excitable nerves and so forth, and it is implausible that the suites of gene products required to support the coordinated tasks of any one cell type, let alone all of them, would arise simply as a consequence of dynamical partitioning (Newman (2020)). Furthermore, the different cell types within an organ (e.g., in the lung, the gas permeable type 1 pneumocytes, the capillary-lining endothelium, and the surfactant-producing type 2 pneumocytes of a lung air sac), need to operate as a unit (Ross et al. (2002)). It is highly unlikely that mathematically determined attractor states of a dynamical system would functionally coordinately in such a fashion (Newman (2020)).

Notwithstanding these caveats concerning modeling cell differentiation into different cell types, in certain cases, dynamical systems have proved an apt modelling approach for the narrower concept of cell state transitions. Specifically, such models can capture aspects of transitions between cell states adjacent to one another in a developmental lineage. Here, the states on either side of the transition have defined functional relationships that depend on different levels of expression of a small number of key factors. Thus, dynamical systems, in the form of Boolean functions or ordinary differential equations (ODEs), have been used to model differentiation in the mammalian red and white blood cell-forming systems, (Chang et al. (2006); Mojtahedi et al. (2016)), regionalization of the gut in the sea urchin larva (Cui et al. (2017)), developmental cell type diversification in insect sensory organs and the nematode vulva (Corson et al. (2017); Corson and Siggia (2017)), and in the morphogenesis of flowers (Alvarez-Buylla et al. (2008)).

Here we describe an example of a dynamical bifurcation leading to incipient differentiation during vertebrate embryogenesis: the formation of precartilage compactions (Barna and Niswander (2007)) or protocondensations (Bhat et al. (2011)) during skeletogenesis of the avian limb. In an earlier paper, we analysed the conditions for the breaking of spatial symmetry in an integro-differential equation model of the two-galectin reactiondiffusion-adhesion network of the developing avian limb (Glimm et al. (2014)). This network, comprising the proteins galectin-1A (Gal-1A) and galectin-8 (Gal-8) and their galactoside-bearing cell surface counterreceptors was experimentally found to mediate both protocondensation morphogenesis and the patterning of the subsequent precartilage condensations (Bhat et al. (2011)). In the present study, we focus only on the intracellular branch of this network, factoring out the cell-cell interaction branch required for spatial pattern formation. This has permitted us to study the dynamics of the transition between the intracondensation and extracondensation states in a reduced ODE system. Experimental evidence indicates that these states represent distinct cellular phenotypes at the biochemical, morphological, and functional levels, and not simply variant packing arrangements of a uniform cell type (Barna and Niswander (2007)). Our mathematical analysis supports this inference, indicating that the two-galectin system exhibits two stable states characterized by high and very low concentrations of the galectins.

Our model is thus an example of a dynamical system with switch-like behavior, arising (in an extended model 
with sigmoidal production terms) from a monostable system via a saddle-node bifurcation. This conceptually simple mechanism has been well-studied as a prototypical way in which molecular switches arise in regulatory networks, both in large-scale models (e.g. Tyson et al. (2001, 2003)) and more abstract geometric models motivated by Waddington's epigenetic landscape analogy (e.g. Corson and Siggia (2017); Ferrell (2012); JutrasDub et al. (2020)). Our model is simple, arising from a reduction of a seven-species model to a three-species system via elimination of "fast" variables, while firmly based on experimentaly well understood interactions. As a consequence, the model is very tractable analytically - we are able to provide an explicit Lyapunov function, for instance - while still being grounded in biological reality.

\section{The model}

\subsection{Derivation}

We first set up a model with seven variables to describe the galectin network. Under the assumption of fast galectin-counterreceptor binding, this then reduces to a system of four coupled ODEs.

The galectin dynamical network is described in the diagram in Figure 1. This is based on experimental data described in Bhat et al. (2011). The associated spatiotemporal partial differential equations model of Glimm et al. (2014) also includes morphogen diffusion and cell adhesion and motion, but not a detailed analysis of the galectin network by itself. There are two freely diffusible, ECM bound protein, Gal-1A and Gal-8. They bind to two different cell-membrane bound counterreceptors. The first of these counterrecptors, which we call Gal-8's counterreceptor, can only bind to Gal-8. This activated the production of Gal-1A. The second, which we call the shared counterrecptor, can bind to both Gal-1A and Gal-8. The binding of Gal-1A to the shared counterrecptor activates both the production of Gal-8 and the production of the shared counterrecptor. The binding of Gal- 8 to the shared counterrecptor has no regulatory effect other than interfering with the action of Gal-1A.

We denote the concentration of unbound shared counterreceptors by $L_{1}$ and $L_{8}$ is the concentration of Gal8's counterreceptor. $G_{1}$ is the concentration of the complex of Gal-1A and the shared counterreceptor, $G_{8}^{8}$ is the concentration of the complex of Gal-8 and its counterreceptor, and $G_{8}^{1}$ is the concentration of the complex of the shared counterreceptor with Gal-8. The concentrations of the two unbound galectins are denoted by $c_{1}$ and $c_{8}$, respectively. Note that these are secreted proteins, so in principle, they can diffuse through the extracellular matrix. Thus in spatially extended systems, galectins provide signals that can coordinate cell behavior spatially. However in the present paper, we are interested in the local properties of the galectin regulation network and its possible states.

Based on experimental evidence and using simple mass action laws in a compartmental model (Figure 1), we can set up the following system of ODEs to describe the dynamics. (These equations were derived in Glimm et al. (2014) from a spatiotemporal model under the assumption of zero cell motility.) 


$$
\begin{aligned}
\frac{d G_{1}}{d t} & =\alpha_{1} c_{1} L_{1}-\alpha_{2} G_{1} \\
\frac{d G_{8}^{8}}{d t} & =\beta_{8,1} c_{8} L_{8}-\beta_{8,2} G_{8}^{8} \\
\frac{d G_{8}^{1}}{d t} & =\beta_{1,1} c_{8} L_{1}-\beta_{1,2} G_{8}^{1} \\
\frac{d L_{1}}{d t} & =\gamma_{1} G_{1}-\gamma_{2} L_{1}-\frac{d G_{1}}{d t}-\frac{d G_{8}^{1}}{d t} \\
\frac{d L_{8}}{d t} & =\delta_{1}-\delta_{2} L_{8}-\frac{d G_{8}^{8}}{d t} \\
\frac{d c_{1}}{d t} & =\nu G_{8}^{8}-\alpha_{1} c_{1} L_{1}+\alpha_{2} G_{1}-\pi_{1} c_{1} \\
\frac{d c_{8}}{d t} & =\mu G_{1}-\beta_{1,1} c_{8} L_{1}+\beta_{1,2} G_{8}^{8}-\beta_{8,1} c_{8} L_{8}+\beta_{8,2} G_{8}^{8}-\pi_{8} c_{8} .
\end{aligned}
$$

We make the assumption that binding of galectins occurs on a faster time scale than production of counterreceptors and galectins. This is expressed by the quasi steady state assumptions

$$
\frac{d G_{1}}{d t}=0, \quad \frac{d G_{8}^{1}}{d t}=0, \frac{d G_{8}^{8}}{d t}=0 .
$$

This makes $G_{1}, G_{8}^{8}$ and $G_{8}^{1}$ effectively functions of the remaining variables, namely

$$
G_{1}(t)=\frac{\alpha_{1}}{\alpha_{2}} c_{1}(t) L_{1}(t), \quad G_{8}^{1}(t)=\frac{\beta_{1,1}}{\beta_{1,2}} c_{8}(t) L_{1}(t) \quad G_{8}^{8}(t)=\frac{\beta_{8,1}}{\beta_{8,2}} c_{8}(t) L_{8}(t) .
$$

The remaining variables then satisfy

$$
\begin{aligned}
\frac{d L_{1}}{d t} & =\left(\gamma_{1} \frac{\alpha_{1}}{\alpha_{2}} c_{1}-\gamma_{2}\right) L_{1} \\
\frac{d L_{8}}{d t} & =\delta_{1}-\delta_{2} L_{8} \\
\frac{d c_{1}}{d t} & =\nu \frac{\beta_{8,1}}{\beta_{8,2}} c_{8} L_{8}-\pi c_{1} \\
\frac{d c_{8}}{d t} & =\mu \frac{\alpha_{1}}{\alpha_{2}} c_{1} L_{1}-\pi_{8} c_{8} .
\end{aligned}
$$

We introduce nondimensional variables $t^{*}, c_{1}^{*}, c_{8}^{*}, L_{1}^{*}, L_{8}^{*}$ via

$$
t=\frac{1}{\pi_{1}} t^{*}, \quad L_{8}=\frac{\delta_{1}}{\pi_{1}} L_{8}^{*}, \quad c_{1}=\frac{\pi_{1} \alpha_{2}}{\gamma_{1} \alpha_{1}} c_{1}^{*}, \quad c_{8}=\frac{\pi_{1}^{3} \beta_{8,2} \alpha_{2}}{\alpha_{1} \beta_{8,1} \gamma_{1} \nu \delta_{1}} c_{8}^{*}, \quad L_{1}=\frac{\pi_{1}^{3} \alpha_{2} \beta_{8,2}}{\alpha_{1} \beta_{8,1} \nu \mu \delta_{1}} L_{1}^{*} .
$$

We define dimensionless parameters

$$
\sigma=\frac{\delta_{2}}{\pi}, \quad \rho=\frac{\gamma_{2}}{\pi_{1}}, \quad \pi=\frac{\pi_{8}}{\pi_{1}} .
$$

Rewriting the system (2.8)-(2.11) with these new dimensionless variables and dropping the stars, the system $(2.8)-(2.11)$ is

$$
\begin{aligned}
\frac{d L_{1}}{d t} & =\left(c_{1}-\rho\right) L_{1} \\
\frac{d L_{8}}{d t} & =1-\sigma L_{8} \\
\frac{d c_{1}}{d t} & =c_{8} L_{8}-c_{1} \\
\frac{d c_{8}}{d t} & =c_{1} L_{1}-\pi c_{8} .
\end{aligned}
$$




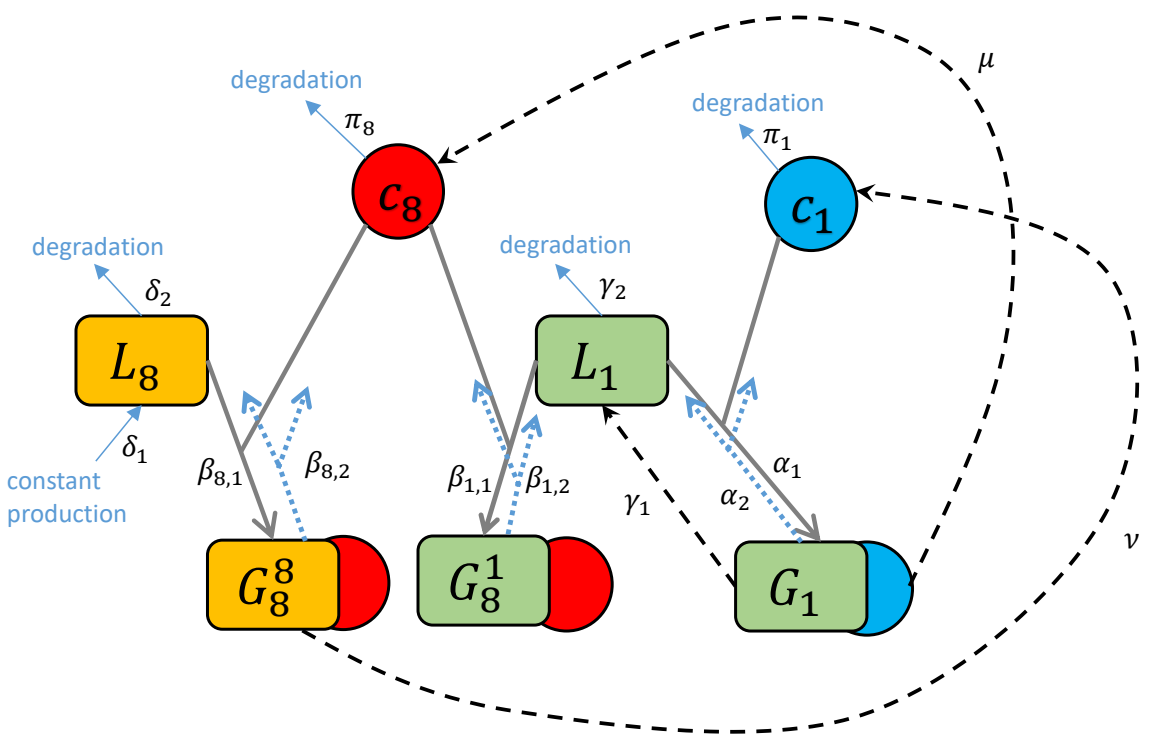

Figure 1: Schematics of the galectin network. Galectins are denoted by $c_{1}$ and $c_{8}$, unbound counterreceptors by $L_{8}$ and $L_{1}$ and the complexes of galectins bound to counterreceptors by $G_{8}^{8}, G_{8}^{1}$ and $G_{1}$. Solid arrows denote reversible binding of galctins and counterreceptors with the unbinding shown by dotted arrows. Dashed arrows denote activation.

This is the central system of this paper. Note that the equation for $L_{8}(t)$ is independent of the other variables and can be solved explicitly as $L_{8}(t)=1 / \sigma+\left(L_{8}(0)-1 / \sigma\right) \exp (-\sigma t)$. In particular, $L_{8}(t) \rightarrow 1 / \sigma$ as $t \rightarrow \infty$. This is why we will also sometimes consider the three variable system where $L_{8}=1 / \sigma$ :

$$
\begin{aligned}
\frac{d L_{1}}{d t} & =\left(c_{1}-\rho\right) L_{1} \\
\frac{d c_{1}}{d t} & =\frac{1}{\sigma} c_{8}-c_{1} \\
\frac{d c_{8}}{d t} & =c_{1} L_{1}-\pi c_{8} .
\end{aligned}
$$

\section{$2.2 \quad$ Parameters}

The system of equations (2.13)-(2.16) contains three parameters $\rho, \sigma$ and $\pi$. All three represent the ratios of degradation constants and that can in principle be estimated based on experimental data for the degradation rates of galectins and membrane-bound receptors. (See Table 1; see also the list of parameters used by Bhat et al. (2019).) A crucial role is the time scale $\hat{t}=1 / \pi_{1}$ of the nondimensionalized time variable $t^{*}$. The half life of galectins is unknown, but likely on the order of several hours (Chen et al. (2014)). Based on the assumption of $\pi_{1} \approx 1$ /day (Bhat et al. (2019)), which corresponds to a half life of Gal-1A of about 16 hours, this gives as the time scale a value of $\hat{t}=1 / \pi_{1} \approx 24$ hours. Likewise, the half-life of counterreceptors has not been measured exactly, but the analysis of 552 human cell-surface glycoproteins yielded a mean half-life of 19.6 hours, with glycoproteins with receptor functions tending to have smaller half-lives than others (Xiao and $\mathrm{Wu}(2017)$ ). This means that the half-lives of galectins and their counterreceptors are likely of the same order of magnitude. Our mathematical results are independent of the ratio of half lives, but for the illustrations, we asume a ratio of 2 . 


\begin{tabular}{cclcc} 
parameter & definition & meaning & est. value & est. plausible range \\
\hline$\rho$ & $\gamma_{2} / \pi_{1}$ & ratio of half-lives of Gal-1A and shared counterreceptor & 2 & $0.2<\rho<20$ \\
$\sigma$ & $\delta_{2} / \pi_{1}$ & ratio of half-lives of Gal-1A and Gal-8's counterreceptor & 2 & $0.2<\sigma<20$ \\
$\pi$ & $\pi_{8} / \pi_{1}$ & ratio of half-lives of Gal-1A and Gal-8 & 1 & $0.1<\pi<10$
\end{tabular}

Table 1: Parameters for the system of equations (2.13)-(2.16). Plausible ranges are within one order of magnitude of the estimated value. Note that due to the chemical similarity of Gal-1A and Gal-8 on the one hand, and of the counterreceptors on the other, it is very plausible to assume that $\pi$ and the ratio $\rho / \sigma$ are both close to unity.

\subsection{Positivity of solutions}

The system (2.13)-(2.16) preserves positivity of solutions, consistent with the fact that negative values for the variables are not biologically meaningful:

Theorem 2.1. Suppose $\left(L_{1}(t), L_{8}(t), c_{1}(t), c_{8}(t)\right)$ is a solution to the system (2.13)-(2.16) with positive initial condition: $L_{1}(0), L_{8}(0), c_{1}(0), c_{8}(0)>0$. Then $L_{1}(t), L_{8}(t), c_{1}(t), c_{8}(t)>0$ for all $t>0$.

Proof. It's clear that $L_{8}(t)$ will be positive for all $t>0$ if $L_{8}(0)$ is positive. Note that $L_{1}\left(t^{*}\right)=0$ for some time $t^{*}$ implies that $L_{1}(t)=0$ for all time $t$. Moreover, by inspection $d c_{1} /\left.d t\right|_{c_{1}=0, c_{8}>0, L_{8}>0}>0$ and $d c_{8} /\left.d t\right|_{c_{1}>0, c_{8}=0, L_{1}>0}>0$, so that neither $c_{1}$ nor $c_{8}$ can become zero when all other variables are positive. The only remaining possibility is that both $c_{1}$ and $c_{8}$ become zero at the same time, but then note that $c_{1}\left(t^{*}\right)=c_{8}\left(t^{*}\right)=0$ for some time $t^{*}$ implies that $c_{1}(t)=c_{8}(t)=0$ for all times $t$.

\section{Phase Space}

\subsection{Analysis}

The system (2.13)-(2.16) has two steady states: besides the (mathematically, though not biologically - see below) trivial one $\left(L_{1}^{(1)}, L_{8}^{(1)}, c_{1}^{(1)}, c_{8}^{(1)}\right)=(0,1 / \sigma, 0,0)$, there is one nontrivial state

$$
\left(L_{1}^{(2)}, L_{8}^{(2)}, c_{1}^{(2)}, c_{8}^{(2)}\right)=(\pi \sigma, 1 / \sigma, \rho, \sigma \rho) .
$$

The trivial steady state is always stable; in fact, its linearization matrix has the four negative eigenvalues $-\rho,-\sigma,-1$ and $-\pi$. The linearization matrix at the nontrivial steady state $\left(L_{1}^{(2)}, L_{8}^{(2)}, c_{1}^{(2)}, c_{8}^{(2)}\right)$ is given by

$$
A=\left(\begin{array}{cccc}
0 & 0 & \pi \sigma & 0 \\
0 & -\sigma & 0 & 0 \\
0 & \sigma \rho & -1 & 1 / \sigma \\
\rho & 0 & \pi \sigma & -\pi
\end{array}\right)
$$

Its characteristic polynomial is given by

$$
P(\lambda)=-(\sigma+\lambda) q(\lambda) \quad \text { with } q(\lambda)=\pi \rho-(1+\pi) \lambda^{2}-\lambda^{3} .
$$

Thus, one of the eigenvalues is always $\lambda_{1}=-\sigma$. The other eigenvalues are the roots of $q(\lambda)$. A straightforward application of the Routh-Hurwitz stability criterion confirms that $q(\lambda)$ has exactly one root with positive real part. The other two roots must have negative real parts. 
Thus the nontrivial steady state is always a saddle with the eigenvalue signature $(+,-,-,-)$. We note that the equation (2.14) can be solved explicitly to give $L_{8}(t)=\left(1-\exp (-\sigma t) / \sigma+L_{8}(0) \exp (-\sigma t)\right)$. It is helpful to set $L_{8}=1 / \sigma$ to obtain a three dimensional phase space that can be visualized. The phase space in Figure 2, typical for this system, is separated into two three-dimensional regions: One region where trajectories converge towards the trivial steady state $\left(L_{1}^{(1)}, c_{1}^{(1)}, c_{8}^{(1)}\right)=(0,0,0)$ and one where trajectories diverge to infinity in all three components. The separatrix is the two-dimensional stable manifold of the saddle point $\left(L_{1}^{(2)}, c_{1}^{(2)}, c_{8}^{(2)}\right)=(\pi \sigma, \rho, \sigma \rho)$. An analogous behavior in two dimensions is displayed in an illustrative simplified system with only two variables in Appendix A; see in particular Figure 8.

This simple analysis indicates that there are two stable states: The trivial steady state, where all concentrations are zero, as well as a second state, in which concentrations grow without bounds. (See Corollary 3.3 for a mathematicl proof that solution curves are in fact either unbounded or converge to one of the two equilibria, the stable trvial one or the saddle.) Biologically, these correspond to the states of mesenchymal cells outside of the protocondensations and within condensations, respectively.

The first state also corresponds to the network state, wherein the Gal-1A and Gal-8 mRNA levels are sparse. This was indeed observed in the cells that are not part of precartilage protocondensations through in situ hybridization (Bhat et al, 2011). Cells that were part of precartilage condensations showed high levels of Gal-1A and Gal-8 mRNA levels and therefore represented the second state.

In the biological context, there invariably are layers of regulation that prevent the concentrations of a network's components from growing out of bounds. The Gal-1A-Gal-8 network, for example, has been shown to be under negative regulation of Notch signaling (Bhat et al. (2019)).

\subsection{Absence of substantial oscillations}

Oscillatory behavior is often found in gene regulatory networks and plays a crucial role for instance in Kaneko's theory of cell differentiation (Suzuki et al. (2011)). Temporal oscillations of the expression rates of the transcription factor Hes1 occur during condensation formation in limb development, where they interact with the galectin regulatory network (Bhat et al. (2019)). It is thus natural to ask whether the system (2.17)-(2.19) can exhibit such oscillatory behavior.

In this section, we prove that this is not the case. In fact, solutions are either unbounded, or they converge to the stable trivial steady state or the saddle point. This follows from the existence of a Lyapunov function:

Theorem 3.1. Consider the function

$$
\mathcal{L}\left(L_{1}, c_{1}, c_{8}\right)=\frac{c_{8}}{c_{1}}+\sigma(\pi+1) \log c_{1}-\frac{1}{\rho} c_{8}+\frac{1}{\rho} L_{1}-\frac{\pi \sigma}{\rho} \log L_{1}-\frac{\pi \sigma}{\rho} c_{1} .
$$

Then $\mathcal{L}\left(L_{1}, c_{1}, c_{8}\right)$ is decreasing along solutions $\left(L_{1}(t), c_{1}(t), c_{8}(t)\right)$ of the system (2.17)-(2.19). Indeed, for $\left(L_{1}(t), c_{1}(t), c_{8}(t)\right)>0$, we have

$$
\frac{d}{d t} \mathcal{L}\left(L_{1}(t), c_{1}(t), c_{8}(t)\right)=-\frac{1}{\sigma}\left(\frac{c_{8}(t)}{c_{1}(t)}-\sigma\right)^{2}
$$

Proof. This follows directly from computing the derivative of $\mathcal{L}$ and substituting the derivatives for $c_{1}, c_{8}, L_{1}$ and $L_{8}$ with the equations (2.17)-(2.19).

Corollary 3.2. There are no non-constant periodic solutions to the system (2.17)-(2.19).

Proof. According to Theorem 2.1, we can assume that $c_{1}(t) \neq 0$ for all $t \in[0, T)$. If $\left(L_{1}(\cdot), c_{1}(\cdot), c_{8}(\cdot)\right)$ is a periodic solution of period $T>0$, then 


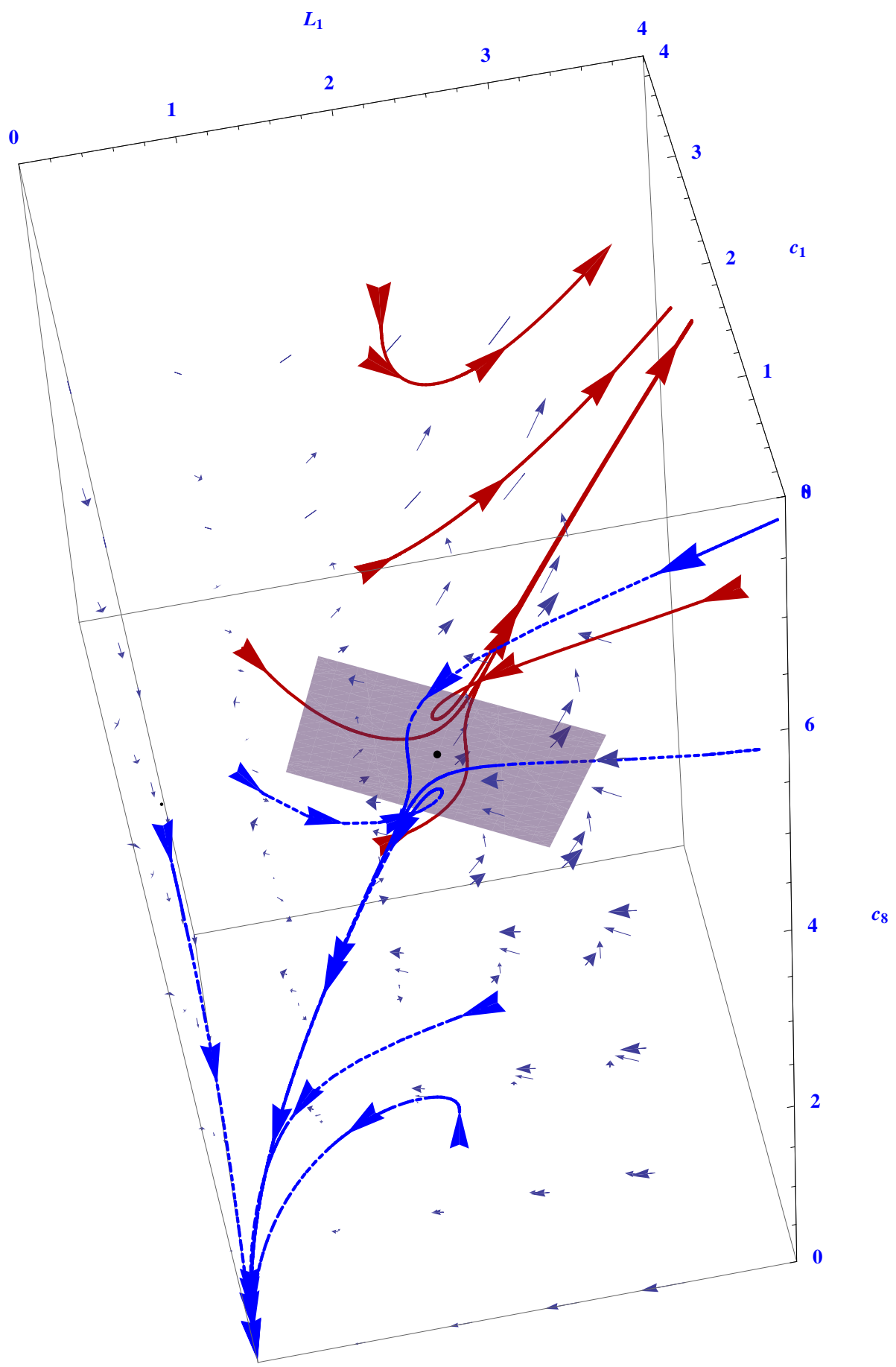

Figure 2: Phase space for the system of equations (2.13)-(2.16) for $\sigma=\rho=2$ and $\pi=1$. Blue dashed trajectories converge to the trivial steady state. Red solid trajectories diverge to infinity. The saddle is shown with tangent space to the stable manifold. 


$$
0=\mathcal{L}\left(L_{1}(T), c_{1}(T), c_{8}(T)\right)-\mathcal{L}\left(L_{1}(0), c_{1}(0), c_{8}(0)\right)=\int_{0}^{T} \mathcal{L}\left(L_{1}(t), c_{1}(t), c_{8}(t)\right) d t=-\frac{1}{\sigma} \int_{0}^{T}\left(\frac{c_{8}(t)}{c_{1}(t)}-\sigma\right)^{2} d t .
$$

It follows that for such a solution we would have $c_{8}(t)=\sigma c_{1}(t)$ for all $t \in[0, T]$. From the second equation, this would imply that $c_{1}(t)=c_{1}(0)$ and $c_{8}(t)=c_{1}(0) \sigma$ for all $t \in[0, T]$. Then the left hand side of the third equation would be zero, implying that $L_{1}(t)=$ const. But then from the first equation we obtain $c_{1}=\rho$, hence $L_{1}=\pi \sigma$. Hence the solution coincides with the unstable stationary point.

Corollary 3.3. If $\left(L_{1}(\cdot), c_{1}(\cdot), L_{1}(\cdot)\right)$ is a bounded solution, then either $\left(L_{1}(\cdot), c_{1}(\cdot), L_{1}(\cdot)\right) \rightarrow(0,0,0)$, or $\left(L_{1}(\cdot), c_{1}(\cdot), L_{1}(\cdot)\right) \rightarrow(\pi \sigma, \rho, \sigma \rho)$ as $t \rightarrow \infty$.

Proof. $\mathcal{L}\left(L_{1}(t), c_{1}(t), c_{8}(t)\right)$ is decreasing on trajectories, so

$$
\mathcal{L}\left(L_{1}(t), c_{1}(t), c_{8}(t)\right) \rightarrow \text { const }<\infty \quad \text { or } \quad \mathcal{L}\left(L_{1}(t), c_{1}(t), c_{8}(t)\right) \rightarrow-\infty
$$

as $t \rightarrow \infty$. In the first case $d \mathcal{L} / d t \rightarrow 0$ as $t \rightarrow \infty$, so $\frac{c_{8}(t)}{c_{1}(t)} \rightarrow \sigma$. This means that $\left(L_{1}(t), c_{1}(t), c_{8}(t)\right)$ approaches the intersection of a level set of $\mathcal{L}\left(L_{1}, c_{1}, c_{8}\right)$ with the plane $c_{8}=\sigma c_{1}$. Since $\left(L_{1}(t), c_{1}(t), c_{8}(t)\right)$ is bounded and there are no periodic orbits according to Corollary 3.2, this implies that $\left(L_{1}(t), c_{1}(t), c_{8}(t)\right)$ must converge to one of the steady states, either $(0,0,0)$ or the saddle point $(\pi \sigma, \rho, \sigma \rho)$ In the second case $c_{1}(t)$ cannot be bounded away from zero. This means $L_{1}(t)$ is not bounded away from zero either. By inspection of the system (2.17)-(2.19), this implies that $\left(L_{1}(t), c_{1}(t), c_{8}(t)\right)$ will eventually lie in the basin of attraction of the nodal sink $(0,0,0)$. Thus $\left(L_{1}(t), c_{1}(t), c_{8}(t)\right) \rightarrow(0,0,0)$ as $t \rightarrow \infty$.

While there are no limit cycles, there can be damped spirals in the vicinity of the saddle point. This is the case for complex eigenvalues:

Proposition 3.4. The saddle point of system (2.13)-(2.16) has a pair of complex eigenvalues if and only if the parameters $\rho$ and $\pi$ satisfy

$$
\rho>\frac{4}{27} \frac{(1+\pi)^{3}}{\pi}
$$

Proof. Consider the characteristic polynomials (3.2) of the linearization matrix (3.1). The discriminant of the third order polynomial $q(\lambda)$ is given by $\Delta=4 \pi(1+\pi)^{3} \rho-27 \pi^{2} \rho^{2}$. Complex eigenvalues exist if and only if $\Delta<0$. This is equivalent to the above inequality.

If the saddle point is a spiral saddle with complex eigenvalue $\lambda$, then the ratio $b=|\operatorname{Im}(\lambda) / \operatorname{Re}(\lambda)|$ is a measure of how tightly the spiral is coiled: Close to the saddle point, the projection of solution curves onto the stable manifold is a spiral whose distance to the saddle decreases by the factor $\exp (-2 \pi / b)$ with each turn. Thus in the limit $b \rightarrow \infty$, the spiral approaches an ellipse, and in the limit $b \rightarrow 0$, it approaches a straight line. A graph of $b=|\operatorname{Im}(\lambda) / \operatorname{Re}(\lambda)|$ as a function of the parameters $\rho$ and $\pi$ shows that $b$ is an increasing function of $\pi$ for fixed $\rho$ (see Figure 3). However, it is bounded from above:

Proposition 3.5. Let $\lambda(\rho, \pi)$ and $\bar{\lambda}(\rho, \pi)$ denote the pair of complex eigenvalues of the saddle point of the system (2.13)-(2.16) which exist for $\rho>\frac{4}{27} \frac{(1+\pi)^{3}}{\pi}$. Then for fixed $\pi$, we have

$$
\lim _{\rho \rightarrow \infty}\left|\frac{\operatorname{Im}(\lambda)}{\operatorname{Re}(\lambda)}\right|=\sqrt{3} \approx 1.732 .
$$

Proof. See Appendix B.

The result shows that there is a lower limit on the tightness of the spiral, implying that from a practical point of view, oscillatory behavior does not play a role for any parameter values of the system (2.13)-(2.16). 


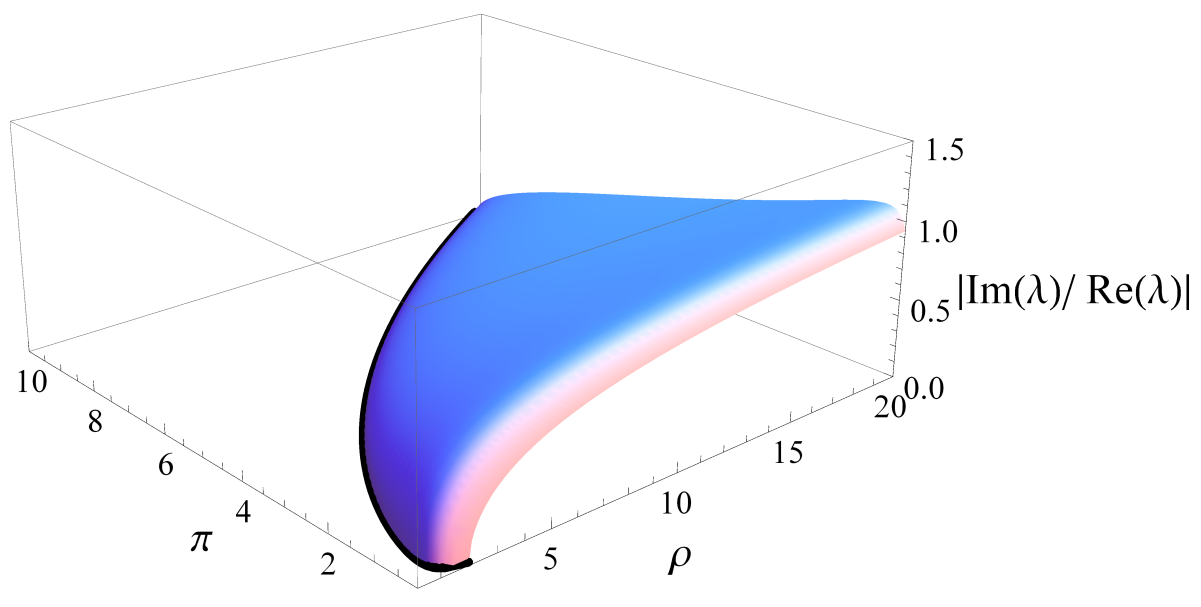

Figure 3: Plot of the ratio - $\operatorname{Im}(\lambda) / \operatorname{Re}(\lambda)$ - of the complex eigenvalue of the saddle point of $(2.13)-(2.16)$ as a function of the parameters $\rho$ and $\pi$.

\subsection{Extension: Sigmoidal production terms}

Note that the system (2.13)-(2.16) does not exhibit bifurcations when the parameters are varied. However, the phase portrait somewhat resembles one of a system with initially one stable node that underwent a saddle-node bifurcation and now has two stable nodes separated by the stable manifold of a saddle point. An analogous role to the second stable node in such a system is played by trajectories which go to infinity in our model, so that we have two possible long term states with attractors of nonzero measure: the trivial steady state and a second one represented by trajectories going to infinity.

The latter is of course unrealistic since exponential growth of concentrations will eventually be slowed by external effects. We thus here extend our model to include such effects. This yields a second stable steady state, which replaces trajectories going to infinity, and also gives a system that can undergo a saddle-node bifurcation.

The possibility of unconstrained growth in the system (2.13)-(2.16) is due to the assumption that the production rates of galectins and counterreceptors are unbounded because of mass action kinetics. A more realistic approach is to assume that these rates have a sigmoidal functional form instead of a linear one. Using standard Michaelis-Menten functions, this yields the following variant of the system (2.13)-(2.16):

$$
\begin{aligned}
\frac{d L_{1}}{d t} & =\left(\frac{c_{1}}{1+\alpha c_{1} L_{1}}-\rho\right) L_{1} \\
\frac{d L_{8}}{d t} & =1-\sigma L_{8} \\
\frac{d c_{1}}{d t} & =\frac{c_{8} L_{8}}{1+\beta c_{8} L_{8}}-c_{1} \\
\frac{d c_{8}}{d t} & =\frac{c_{1} L_{1}}{1+\gamma c_{1} L_{1}}-\pi c_{8},
\end{aligned}
$$

where $\alpha, \beta$ and $\gamma$ are additional (nonnegative) parameters. Note that the model (2.13)-(2.16) corresponds 
to $\alpha=\beta=\gamma=0$. For fixed $\alpha, \beta$ and $\gamma$, there are pairs of solutions of the steady state equations given by

$$
\begin{aligned}
\left(\bar{L}_{1}\right)_{+/-} & =\frac{\pi \alpha \rho \sigma-\beta \rho-\pi \gamma \rho \sigma+1 \pm R(\alpha, \beta, \gamma \rho, \pi, \sigma)}{2 \alpha \rho} \\
\left(\bar{L}_{8}\right)_{+/-} & =\frac{1}{\sigma} \\
\left(\bar{c}_{1}\right)_{+/-} & =\frac{-\pi \alpha \rho \sigma+\beta \rho+\pi \gamma \rho \sigma+1 \pm R(\alpha, \beta, \gamma \rho, \pi, \sigma)}{2(\beta+\pi \gamma \sigma)} \\
\left(\bar{c}_{8}\right)_{+/-} & =\frac{-\pi \alpha \rho \sigma-\beta \rho+\pi \gamma \rho \sigma+1 \pm R(\alpha, \beta, \gamma \rho, \pi, \sigma)}{2 \pi(\alpha \beta \rho-\beta \gamma \rho+\gamma)}
\end{aligned}
$$

where

$$
R(\alpha, \beta, \gamma \rho, \pi, \sigma)=\sqrt{(\pi \rho \sigma(\gamma-\alpha)+\beta \rho+1)^{2}-4 \rho(\beta+\pi \gamma \sigma)} .
$$

Here the subscript "+" denote the larger branch and "-" the smaller branch. This system undergoes a saddlenode bifurcation when $R(\alpha, \beta, \gamma \rho, \pi, \sigma)$ switches from imaginary to real values such that both branches of are positive. The larger branch $\left(\left(\bar{L}_{1}\right)_{+},\left(\bar{c}_{1}\right)_{+},\left(\bar{c}_{8}\right)_{+}\right)$is stable, the lower branch $\left(\left(\bar{L}_{1}\right)_{-},\left(\bar{c}_{1}\right)_{-},\left(\bar{c}_{8}\right)_{-}\right)$is a saddle. An example of a bifurcation diagram is shown in Figure 4.

Note that the simpler "mass action" system (2.13)-(2.16) can be formally obtained from the system (3.4)(3.7) via setting $\alpha=\beta=\gamma=\varepsilon$ and taking the limit $\varepsilon \rightarrow 0$. Indeed, one checks that with $\alpha=\beta=\gamma=\varepsilon$, one gets

$$
\lim _{\epsilon \rightarrow 0}\left(\left(\bar{L}_{1}\right)_{-},\left(\bar{c}_{1}\right)_{-},\left(\bar{c}_{8}\right)_{-}\right)=(\pi \sigma, \rho, \rho \sigma)
$$

and

$$
\lim _{\epsilon \rightarrow 0}\left(\left(\bar{L}_{1}\right)_{+},\left(\bar{c}_{1}\right)_{+},\left(\bar{c}_{8}\right)_{+}\right)=(\infty, \infty, \infty) .
$$

One thus recovers the "infinite" state and the saddle of the system (2.13)-(2.16) as the limits of the stable node and the saddle point of the system (3.4)-(3.7), respectively.

\section{Experimental data}

In contrast to a spectrum of biochemical states with progressively changing galectin expression, our model predicts the concurrent emergence of two distinct dynamical cell states: one with high galectin production (intra-protocondensation cells) and the other with low galectin production rates (extra-protocondensation cells) as a consequence of the reaction kinetics of the galectin network.

For experimental validation, we took the two complementary approaches described in the sections below to determine whether the cells within protocondensations differ qualitatively in their properties from those outside of protocondensations.

\subsection{High resolution fluorescence imaging of Gal-1A shows higher per-cell Gal-1A concentration within forming condensations.}

For experimental corroboration, we focused on the differences between condensing and noncondensing mesenchymal cells within high density micromass cultures of chick limb buds. Using fluorescent microscopy of micromasses stained for Gal-1A (with counterstaining for DNA using DAPI), we found that cells within forming protocondensations had significantly higher levels of Gal-1A compared to cells outside. However, protocondensations were also the locales within the micromass where the cell density was highest. Therefore, we asked whether the relatively higher Gal-1 staining within protocondensations was just a density-dependent optical artifact. 


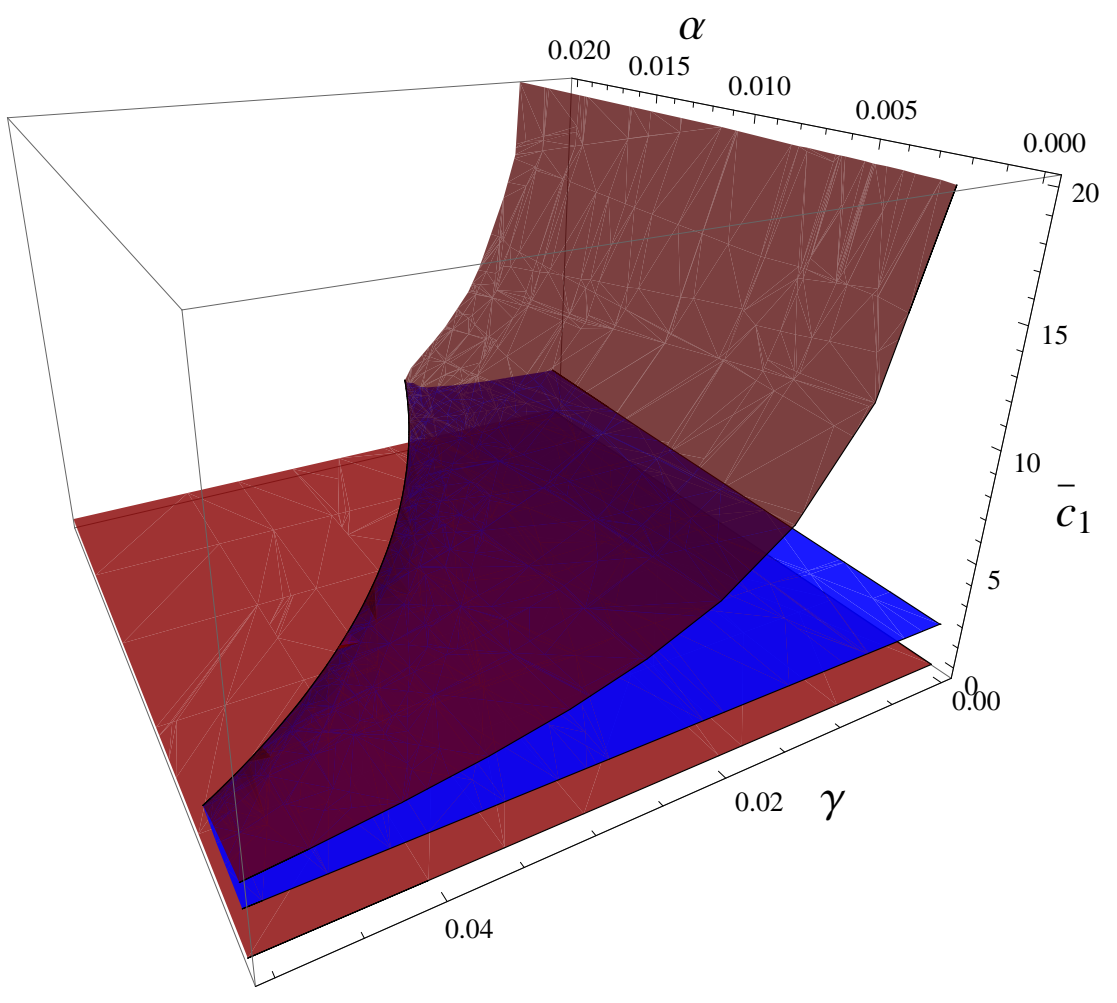

Figure 4: Bifurcation diagram for the equations (3.4)-(3.7) for $\pi=1, \sigma=\rho=2$ and $\beta=0.01$. The vertical axis shows the steady state $\bar{c}_{1}$. Stable nodes are shown in red. Saddles are shown in blue. Note the saddle-node bifurcations along a curve in $\alpha-\gamma$ space. 

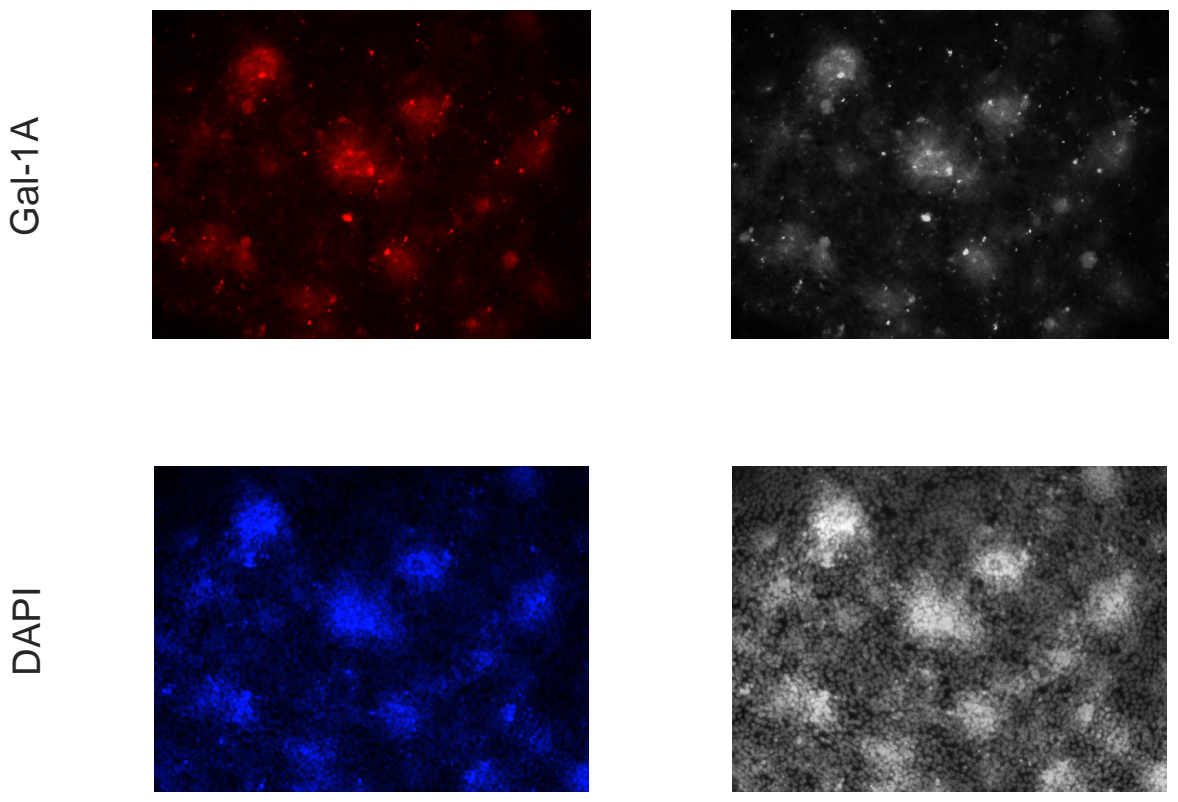

Figure 5: High resolution immunofluorescence images of precartilage condensations imaged for Gal-1A (top row) and DNA (using DAPI (bottom row)). Left column: Original images. Right column: grayscale intensity counterparts of the left column images.

To answer this, we plotted the mean intensity of Gal-1A signal against the intensity of the DAPI signal, yielding a diagram that approximates Gal-1A concentration as a function of cell density (Figure 6). The resulting graph was convex and a least squares approximation yielded an almost exactly quadratic curve. This indicated that the Gal-1A concentration's increase was higher than could be explained by an increase in density of cells expressing equal amounts of Gal-1A. Therefore, the levels of Gal-1A concentration per cell was higher within protocondensations than outside them.

\subsection{Single cell tracking shows qualitative differences in cell motion between intra- condensation and extra-condensation cells.}

We also investigated the motility dynamics of single mesenchymal cells at the initiation of protocondensation formation in vitro using cell-resolved, video-tracking techniques (Figure 7). To record the 480 minutes during ${ }_{266}$ which the protocondensations emerged, we processed 25 successive images taken at 20 minute intervals. (We 267 took time-lapse images at intervals of 2 minutes at a resolution of 640X480 pixels and chose every tenth image ${ }_{268}$ for processing. See movie file M1 in Supplemental Materials.) During the observation period, cells in the 269 monolayer moved, changed their relative positions, and deformed continuously. In each image, we traced 270 condensation centers by determining the cell-substratum attachment area for each cell, smoothing this function ${ }_{271}$ over the whole image and then determining the contour that corresponded to the mean cell-substratum area. 272 Of the 92 cells we tracked in the image set, 46 stayed exclusively inside the condensation center (i.e., within ${ }_{273} 57 \mu \mathrm{m}$ of the center), whereas 33 cells remained exclusively outside. The centers of mass of 13 cells crossed 274 this boundary at least once in either direction during the $8 \mathrm{~h}$ of filming. The velocity statistics of the cells 275 residing exclusively inside and outside the forming condensation are shown in Table 2 . The mean speed of 276 cells exclusively inside condensations exceeded that of cells eclusively inside condensations, and the same held 


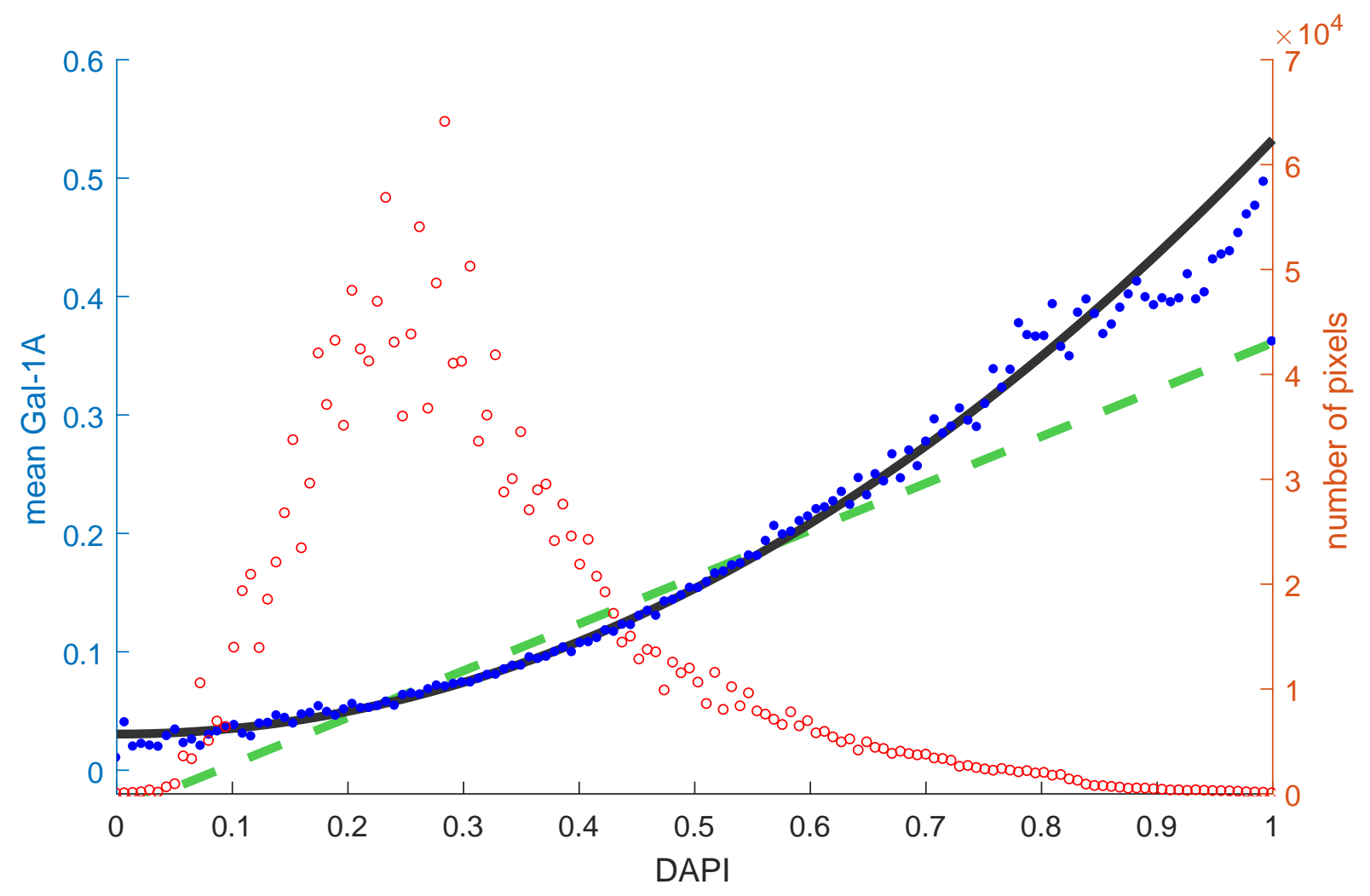

Figure 6: Mean intensity of the grayscale Gal-1A image as a function of the intensity of DAPI. Values are scaled from 0 (completely black pixels) to 1 (white pixels of maximum intensity). Red dots indicate the number of pixels for each DAPI intensity, showing that most pixels have an intensity between about 0.1 and 0.5. Green dashed line: linear regression line, weighted by number of pixels, given by $C G-1 A=-0.034623+0.3956 D A P I$. Gray line: Nonlinear least squares regression of the form $-1 A=a+b D A P I^{c}$ weighted by number of pixels. Best-fit parameters are $a=0.030523, b=0.50199, c=2.0298$. 

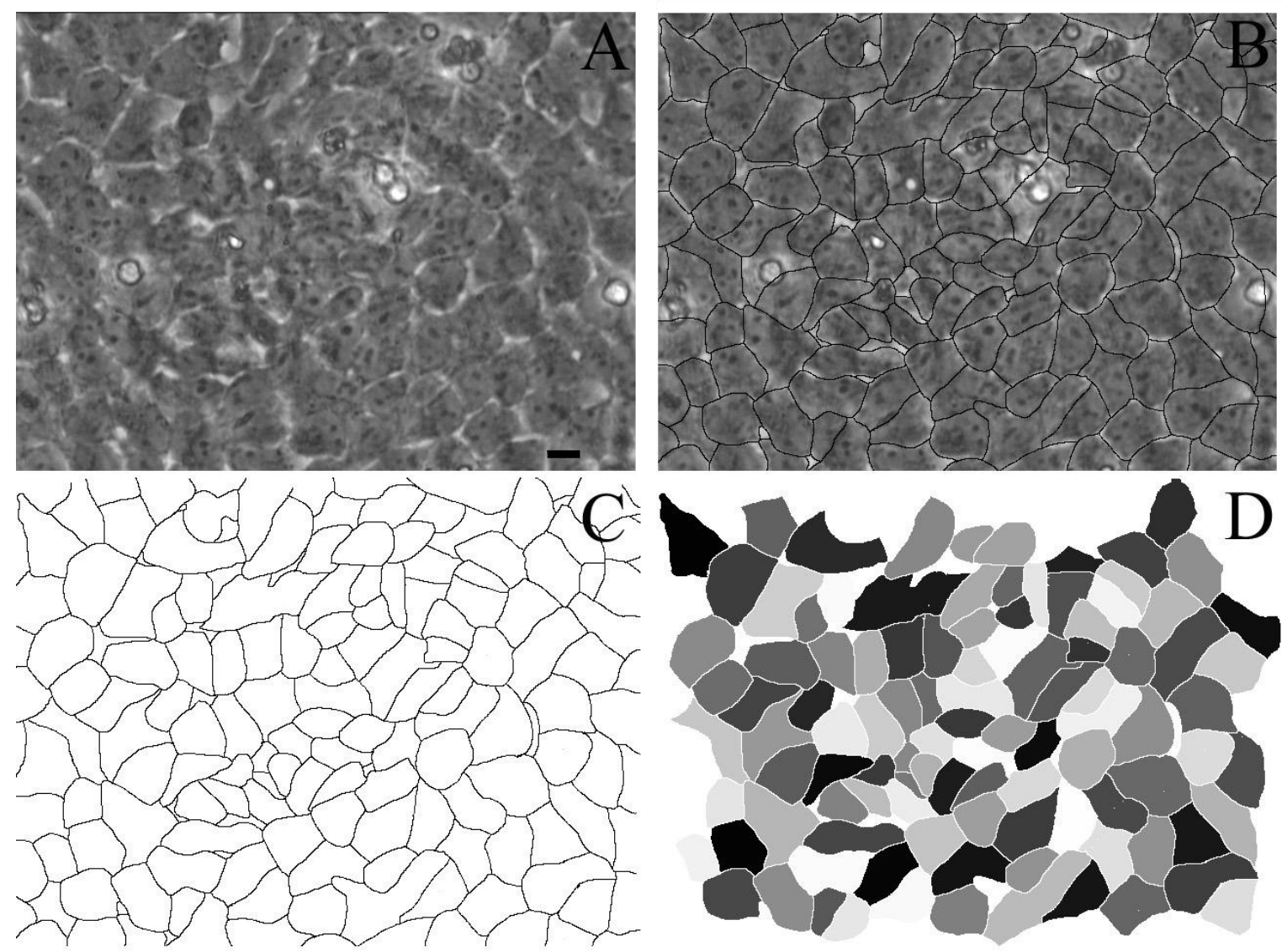

Figure 7: Image digitization. A, Original image: a single frame from the movie. B, Image with boundaries drawn by hand in Photoshop. C, Boundaries of tracked cells. D, Digitized image with gray-level-labeled cells. The scale bar in A represents a length of $10 \mu \mathrm{m}$.

true for the median value. (A two-tailed z-test to test the significance of the difference of the means yielded a $p$-value of approximately $10^{-4}$.) A total of 615 out of the 1104 measured speeds for cells staying exclusively inside the condensation center (or $55.71 \%$ ) were greater than the median value $(0.0867 \mu \mathrm{m} / \mathrm{min}$ ) of the speeds of cells remaining outside the condensation center.

We characterized the linear dimension and number of cells in the observed precartilage condensation and found that interior cells in this region had smaller areas of contact with their substratum than peripheral cells. While cells inside and far outside the forming condensation have random speeds and directions of motion, cells in the periphery of a condensation tend to move towards the center of the condensation (see Figure S1 in the Supplementary Material). This is consistent with a hypothetical haptotaxis mechanism, not intrinsically directed but based on random cell movement with preferntial sticking to increasingly adhesive substrata. In such a model, there is no qualitative difference between intracondensation and extracondensation mesenchymal cells.

However, contrary to the predictions of the haptotaxis model, we found that cells within the forming condensation move significantly faster than cells in the periphery. Indeed, the equating of enhanced cell-substratum binding to stickiness in the conventional version of haptotaxis would suggest that cells should slow down as they are incorporated into condensations. The fact that the opposite happens points to a qualitative behavioral difference between intracondensation and extracondensation cells. 


\begin{tabular}{|c|c|c|}
\hline & Inside & Outside \\
\hline Number of cells & 46 & 33 \\
\hline mean value $(\mu \mathrm{m} / \mathrm{min})$ & 0.1141 & 0.1012 \\
\hline standard deviation & 0.0761 & 0.062 \\
\hline median $(\mu \mathrm{m} / \mathrm{min})$ & 0.0941 & 0.0867 \\
\hline
\end{tabular}

Table 2: Comparison of velocities of cells residing exclusively inside and outside precartilage condensation.

\section{Discussion}

In this paper, we have investigated a core dynamical switch, embedded in the chicken limb buds skeletal pattern-forming network, that controls the transition between two lineage-adjacent cell states: uncondensed and protocondensed (Bhat et al. (2011)) precartilage mesenchyme. The phase space of the dynamical system consists of two regions, one in which trajectories converge to a stationary state and the other in which trajectories are unbounded. These two regions are separated by the stable manifold of a saddle point. The two fates convergence to a steady state or growth without bound - correspond biologically to cells within a protocondensation and cells outside this region, and are marked, respectively (among other differences) by very high and very low levels of Gal-1A. If the assumption of linear production of galectins and counterreceptors is replaced by sigmoidal production terms, the region of unbounded trajectories instead becomes the basin of attraction for a second steady state, and thus the system then displays typical bistability attained via a saddle-node bifurcation (Strogatz (1994); Griffith (1971); Graham et al. (2010)) with a high and a low state, see section 3.3.

In addition to determining the concentrations of Gal-1A and Gal-8, Eqs. 2.13-2.16, the defining dynamic system for the cell state transition, specify the concentrations of each of the two galectins bound to their receptors (one shared by the two galectins and a Gal-8-specific one) as well as the concentrations of the receptors themselves. The two biologically relevant states reflect differences in those factors as well. But while this set of molecules are controlling variables for the transition, they do not exhaustively define the intra- and extraprotocondensed or compacted states, which include differences in levels or activities of BMP receptor 1b, the transcription factor Sox9 (Barna and Niswander (2007)) and TGF- $\beta$ and responsive nuclear factors that in the following hours will lead to expression of the fibronectin gene as definitive mesenchymal condensations are consolidated (Leonard et al. (1991)).

While the cell-state switch functions as part of a reaction-diffusion-adhesion network in the embryonic limb bud and high-density mesenchymal cell cultures derived from it, extracting it mathematically from this context allows us to ascertain some of its important features. First, however, limitations of such an operation must be acknowledged. The mathematically isolated core switching network from the model of Glimm et al (2014) predicts that the Gal-1A concentration grows without bounds or goes to zero in the intra- and extraprotocondensation domains. These nonbiological outcomes are artifacts of the isolation of a portion of the full network, in simulations of which realistic values of the galectins are predicted (Glimm et al. (2014); Bhat et al. (2019)). Moreover, replacing the linear production rates for the galectin and counterreceptors by sigmoidal ones, which incorporates that maximum production rates, leads to standard bistability between two cell states (see section 3.3).

More usefully, we note that attractor states of a given dynamical system are not necessarily conserved when components of the system can diffuse (Krause et al. (2018)), or even when identical copies of the system interact with each other (Kaneko and Yomo (1999)). It was therefore unclear before the present study whether or not the foci of high Gal-1A accumulation surrounded by regions of low abundance of this protein predicted by the full reaction-diffusion-adhesion system (Glimm et al. (2014)) were manifestations of cell-cell adhesion or 
communication. Our conclusion from the analysis here that the switch is, in fact, cell-autonomous suggests that it could have been a primitive feature of skeletogenic mesenchymal cells not dependent on fine-tuned, tissue-wide interactions. This, in turn, corresponds to evolutionary scenarios in which variously distributed nodules and plates of endoskeletal tissue arose in the fins of ancestral chordates prior to the stereotypical patterning systems seen in the appendages of sarcopterygian fishes, and particularly tetrapods (Newman et al. (2018)).

Another significant finding is that Gal-1 production is not susceptible to being thrown into an oscillatory state within the biologically plausible region of state space we have explored. This is consistent with the results of a previous study in which a periodic function representing the experimentally ascertained oscillatory behavior of the transcriptional regulator Hes1 was incorporated into the full reaction-diffusion-adhesion system (Bhat et al., 2019). Notwithstanding the potential entrainment of Gal-1 synthesis by its functional dependence on the cell density, which is dependent on periodically varying Hes1 activity by our hypothesis (see Eqs. 1-4 of Bhat et al. (2019)), the accumulation of the galectins increases monotonically over the first $36 \mathrm{~h}$ of development in vitro (Bhat et al., 2011) and under a range of simulation conditions with the Hes1-enhanced model (Bhat et al. (2019)).

While Hes1 oscillations and their synchronization appear to play an important role in the spatial regularity and refinement of the boundaries between condensed and non-condensed mesenchyme in the developing limb (Bhat et al. 2019), the circuitry described here that robustly keeps the production of Gal-1A from becoming periodic is advantageous in a developmental mechanism since the opposite would lead to discontinuities in the spatial pattern (see Glimm et al., 2014).

We characterized the relation between cell density and Gal-1A distribution to confirm another predicted feature of the core switching circuit. In a previous study, we showed that the formation pattern of a patterned distribution of Gal-1A does not occur in the absence of the advection component, i.e., movement of cells up the Gal-1A adhesion gradient, of the full two-galectin reaction-diffusion-adhesion network (Glimm et al. (2014)). That is, the pattern-forming mechanism is morphodynamic in the sense of requiring cell or tissue rearrangement concurrently with the chemical signaling that drives the rearrangement (Salazar-Ciudad et al. (2003)). This raised the possibility that the elevated Gal-1A in protocondensations was tied, in the living system, to the elevated cell density at these sites. However, our quantitative analysis shows that, as implied by the inherent properties of the core circuit, which lacks not only cell movement but even a cell-cell adhesive function, Gal-1A concentration is higher on a per cell basis inside than outside protocondensations (Fig. 7). Finally, our tracking and analysis of motile behaviors within and outside the protocondensations indicate that these populations are true differentiated states. That is, they do not differ simply by the concentrations of the molecular species controlling their partitioning. One hallmark of cell differentiation is the recruitment and exaggerated expression of ancestral cell functions so as to set a population of cells apart from those spatially contiguous to them and adjacent to them in a developmental lineage (Newman (2020)). In the case described here, the incipient functional differentiation represented by the increased randomly oriented velocity of the protocondensed cells is clearly transient: the cell velocity in definitive fibronectin-rich condensations, and certainly in the terminally differentiated cartilage that arises from them will be much reduced, tending to zero. But during early stages of skeletal development, it may be an important factor in stabilizing the patterning of the initial primordia.

This suggests, in turn, that the earliest determinants of limb skeletal pattern in the chicken, Gal-1A and Gal-8, do not directly induce skeletal tissue, cartilage or bone, or even provisional versions of these supportive tissues. Moreover, they do not simply mark out the domains that will subsequently differentiate into these tissues. Rather, development, at least in this case, may occur in steps, with intermediates having specific functions (e.g., enhanced motility in primordia that will later solidify) with no obvious or logical relation to the ultimate one. This is an aspect of development, if it proves more general, with evolutionary implications warranting further exploration. 


\section{Methods}

\subsection{Cell tracking experiment (section 4.2) Cell Culture}

We obtained fertile white Leghorn chicken eggs from Avian Services, Inc. (Frenchtown, NJ). We cut developing legs at Hamburger-Hamilton stage 25 (Hamburger and Hamilton, 1952) $0.3 \mathrm{~mm}$ from the distal end of the limb bud. We used the tips, the mesodermal component of which consists almost entirely of precartilage cells (Newman et al., 1981; Brand et al., 1985), to prepare cells for culture. Cultures were prepared essentially as described (Downie and Newman, 1994). Briefly, we dissociated the cells in 1\% trypsin-EDTA (Sigma), filtered them through Nytex 20- $\mu$ m mono-filament nylon mesh (Tetko, Briarcliff Manor, NY), and resuspended them at $2.0 \times 10^{7}$ cells $/ \mathrm{ml}$ in defined medium (DM, Paulsen and Solursh, 1988) containing $10 \%$ fetal bovine serum. Filtration removed most of the limb-bud ectoderm, which remained in sheets after trypsinization. We deposited one to three cell spots $\left(10 \mu \mathrm{l}\right.$ each, containing $2.0 \times 10^{5}$ cells) in a Falcon $60 \mathrm{~mm}$ tissue-culture dish (Falcon No. 351008) and allowed the cells to adhere to the dish at $38.5^{\circ} \mathrm{C}$ in a $5 \% \mathrm{CO}_{2}$ atmosphere for 15 min before flooding the dish with serum-free DM. Although the initial cell plating density is above confluency, adding the medium disperses cells not attached to the substratum, resulting in a tightly-packed monolayer. We returned the cell cultures to the incubator for 24 hours, then sealed the lids of the culture dishes with Parafilm to prevent dehydration during time-lapse photomicrography.

\section{Immunofluorescence}

High density micromass cultures were fixed with ice-cold 100\% methanol, washed with PBS and permeabilized with $0.02 \%$ Triton X-100 for 10 min. The cultures were incubated with affinity-purified polyclonal rabbit antiCG-1A antibody for $2 \mathrm{~h}$ at room temperature, followed by incubation with DyLight 594-conjugated secondary goat anti-rabbit antibody for $1 \mathrm{~h}$. Following counterstaining of DNA with DAPI, the cultures were imaged with an inverted Zeiss IM35 epifluorescence microscope with appropriate filters and a high-magnification objective (63 $\mathbf{r} A \sim$ oil immersion lens). Post image acquisition, the images were grayscaled and intensity measured using Matlab.

\section{Microscopy and Image Acquisition}

We placed the culture dish in a temperature-controlled Peltier warming device, maintained at $38.5 \mathrm{deg} \mathrm{C}$ on the stage of a Zeiss IM35 inverted microscope equipped with a $32 \times$ Planacromat phase-contrast objective. Warm air blown across the dish lid prevented condensation on the lids inner surface. A timer box turned the microscopes light source on and off in synchrony with the computer-controlled camera to minimize cells exposure to light. We took time-lapse images at intervals of 2 minutes at a resolution of $640 \times 480$ pixels. Of our eight experimental plates, three developed condensations near the center of the microscopes field of view and therefore permitted quantitative study. We chose one of these cultures for detailed analysis. The others developed at the same rate and had the same general morphology as the one we analyzed. We collected more than 350 individual images of the selected culture. Cells in frames 241-350 increasingly defocused as differentiation to cartilage changed cell shapes and the culture thickened. In these frames we could not track individual cells, especially in the condensation center, so we processed and analyzed only frames 1-240. 


\section{Image Processing and Data Analysis for cell-tracking experiments}

The resolution of the images was $0.285 \mu \mathrm{m} /$ pixel, sufficient to track the $10 \mu \mathrm{m}$ diameter cells center-of-mass motions and deformations. With the aid of ImageJ software, which can play a sequence of images forward and backward, we hand-traced the boundaries between individual cells using Photoshop. Cells moved much less than one cell radius between frames, so we could unambiguously identify which cells in each image corresponded to the same experimental cell. We then used Photoshop and Matlab to assign a district gray level to each experimental cell for display and tracking (Fig.7).

For the statistical analysis, we digitized every tenth image, processing 25 images. In the image set the interval between two successive processed images is $20 \mathrm{~min}$ and the total duration is $480 \mathrm{~min}$.

We labeled only cells lying completely within the field of view during the entire time series. We did not label or use cells which crossed the boundary of the field of view or which moved in or out of the field of view during the series. We observed 27 cell divisions during the 760 min time-lapse series in a population of about 145 cells; 8 of 92 labeled cells divided during the first $480 \mathrm{~min}$. When a cell divided, we assigned to one of its daughter cells its mother cells gray level and used it in our statistical measurements. We assigned the other a new gray level and omitted it from our statistical measurements. By these criteria, the image set has 92 cells.

We used Matlab for image processing and data analysis. We recorded two-dimensional cell surface areas, which we interpreted as areas of contact with the underlying substratum, for all labeled cells. We used the coordinates of the center of mass $\vec{r}_{i}=\left(x_{i}, y_{i}\right)$ to analyze cell displacements. We recorded the mean-squared displacement, $\operatorname{MSD}(\mathrm{t})=\left\langle\Delta \mathrm{r}_{\mathrm{i}}(\mathrm{t})^{2}\right\rangle=\left\langle\left|\tilde{\mathrm{r}}_{\mathrm{i}}(\mathrm{t})-\tilde{\mathrm{r}}_{\mathrm{i}}(0)\right|^{2}\right\rangle$, the average distance a cells center of mass traveled. We approximated the velocity of cells at time $t$ as $\vec{v}_{i}(t)=\left(\vec{r}_{i}(t)-\vec{r}_{i}(t-1)\right) / \Delta t$, where $\Delta t$ is $20 \mathrm{~min}$. We also measured the autocorrelation of velocities: $C(t)=\left\langle\vec{v}_{i}\left(t_{0}+t\right) \cdot \vec{v}_{i}\left(t_{0}\right)\right\rangle /\left\langle\left|\vec{v}\left(t_{0}\right)\right|^{2}\right\rangle \mid$, which quantifies the time scale over which cells maintained the magnitude and direction of their velocities.

\section{Acknowledgements}

B.K. was partially supported by the National Science Centre grant 2016/21/B/ST1/03071. R.B. acknowledges support from DBT [BT/PR26526/GET/119/92/2017] and the Wellcome Trust/DBT India Alliance Fellowship/Grant [Grant Number IA/I/17/2/503312]. Prof. James Glazier participated in the analysis presented in Table 2 .

\section{References}

E. R. Alvarez-Buylla, A. Chaos, M. Aldana, M. Bentez, Y. Cortes-Poza, C. Espinosa-Soto, D. A. Hartasnchez, R. B. Lotto, D. Malkin, G. J. Escalera Santos, and P. Padilla-Longoria. Floral morphogenesis: stochastic explorations of a gene network epigenetic landscape. PloS one, 3(11):e3626, 2008. ISSN 1932-6203. doi: 10.1371/journal.pone.0003626. URL https://europepmc.org/articles/PMC2572848.

M. Barna and L. Niswander. Visualization of Cartilage Formation: Insight into Cellular Properties of Skeletal Progenitors and Chondrodysplasia Syndromes. Developmental Cell, 12(6):931-941, June 2007. ISSN 1534-5807. doi: 10.1016/j.devcel.2007.04.016. URL http://www.sciencedirect.com/science/article/pii/S1534580707001773.

R. Bhat, K. M. Lerea, H. Peng, H. Kaltner, H. J. Gabius, and S. A. Newman. A regulatory network of two galectins mediates the earliest steps of avian limb skeletal morphogenesis. BMC Dev. Biol., 11:6, 2011. 


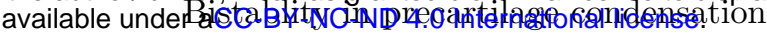

R. Bhat, T. Glimm, M. Linde-Medina, C. Cui, and S. A. Newman. Synchronization of Hes1 oscillations coordinates and refines condensation formation and patterning of the avian limb skeleton. Mechanisms of Development, 156:41-54, Apr. 2019. ISSN 0925-4773. doi: 10.1016/j.mod.2019.03.001. URL http://www.sciencedirect.com/science/article/pii/S0925477318301266.

H. H. Chang, P. Y. Oh, D. E. Ingber, and S. Huang. Multistable and multistep dynamics in neutrophil differentiation. BMC Cell Biology, 7(1):11, Feb. 2006. ISSN 1471-2121. doi: 10.1186/1471-2121-7-11. URL https://doi.org/10.1186/1471-2121-7-11.

C. Chen, C. A. Duckworth, B. Fu, D. M. Pritchard, J. M. Rhodes, and L.-G. Yu. Circulating galectins -2, -4 and -8 in cancer patients make important contributions to the increased circulation of several cytokines and chemokines that promote angiogenesis and metastasis. British Journal of Cancer, 110(3):741-752, Feb. 2014. ISSN 0007-0920. doi: 10.1038/bjc.2013.793. URL https://www.ncbi.nlm.nih.gov/pmc/articles/PMC3915140/.

F. Corson and E. D. Siggia. Gene-free methodology for cell fate dynamics during development. eLife, 6:e30743, Dec. 2017. ISSN 2050-084X. doi: 10.7554/eLife.30743. URL https://doi.org/10.7554/eLife.30743. Publisher: eLife Sciences Publications, Ltd.

F. Corson, L. Couturier, H. Rouault, K. Mazouni, and F. c. Schweisguth. Self-organized Notch dynamics generate stereotyped sensory organ patterns in Drosophila. Science (New York, N. Y.), 356(6337), 2017. ISSN 1095-9203. doi: 10.1126/science.aai7407.

M. Cui, E. Vielmas, E. H. Davidson, and I. S. Peter. Sequential Response to Multiple Developmental Network Circuits Encoded in an Intronic cis-Regulatory Module of Sea Urchin hox11/13b. Cell Reports, 19(2):364-374, 2017. ISSN 2211-1247. doi: 10.1016/j.celrep.2017.03.039.

J. E. Ferrell. Bistability, bifurcations, and Waddington's epigenetic landscape. Current biology: CB, 22(11): R458-466, June 2012. ISSN 1879-0445. doi: 10.1016/j.cub.2012.03.045.

C. Furusawa and K. Kaneko. A dynamical-systems view of stem cell biology. Science, 338(6104):215-217, Oct 2012.

T. Glimm, R. Bhat, and S. A. Newman. Modeling the morphodynamic galectin patterning network of the developing avian limb skeleton. J. Theor. Biol., 346:86-108, Apr 2014.

D. K. Goode, N. Obier, M. S. Vijayabaskar, M. Lie-A-Ling, A. J. Lilly, R. Hannah, M. Lichtinger, K. Batta, M. Florkowska, R. Patel, M. Challinor, K. Wallace, J. Gilmour, S. A. Assi, P. Cauchy, M. Hoogenkamp, D. R. Westhead, G. Lacaud, V. Kouskoff, B. Gottgens, and C. Bonifer. Dynamic Gene Regulatory Networks Drive Hematopoietic Specification and Differentiation. Dev. Cell, 36(5):572-587, Mar 2016.

T. G. W. Graham, S. M. A. Tabei, A. R. Dinner, and I. Rebay. Modeling bistable cellfate choices in the Drosophila eye: qualitative and quantitative perspectives. Development (Cambridge, England), 137(14):2265-2278, July 2010. ISSN 0950-1991. doi: 10.1242/dev.044826. URL https://www.ncbi.nlm.nih.gov/pmc/articles/PMC2889600/.

J. S. Griffith. Mathematical neurobiology: an introduction to the mathematics of the nervous system. Academic Press, London, New York, 1971. ISBN 978-0-12-303050-4. Open Library ID: OL5758380M.

S. Huang. The molecular and mathematical basis of Waddington's epigenetic landscape: a framework for post-Darwinian biology? Bioessays, 34(2):149-157, Feb 2012. 


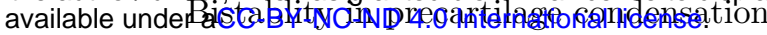

S. Huang, G. Eichler, Y. Bar-Yam, D. E. Ingber, and D. E. Ingber. Cell fates as high-dimensional attractor states of a complex gene regulatory network. Phys. Rev. Lett., 94(12):128701, Apr 2005.

L. Jutras-Dub, E. El-Sherif, and P. François. Geometric models for robust encoding of dynamical information into embryonic patterns. eLife, 9, Aug. 2020. ISSN 2050-084X. doi: 10.7554/eLife.55778.

K. Kaneko and T. Yomo. Isologous diversification for robust development of cell society. J. Theor. Biol., 199 (3):243-256, Aug 1999.

S. A. Kauffman. Metabolic stability and epigenesis in randomly constructed genetic nets. J. Theor. Biol., 22 (3):437-467, Mar 1969.

A. D. Keller. Specifying epigenetic states with autoregulatory transcription factors. Journal of Theoretical Biology, 170(2):175-181, Sept. 1994. ISSN 0022-5193. doi: 10.1006/jtbi.1994.1177.

A. L. Krause, A. M. Burton, N. T. Fadai, and R. A. Van Gorder. Emergent structures in reaction-advectiondiffusion systems on a sphere. Physical Review E, 97(4):042215, Apr. 2018. doi: 10.1103/PhysRevE.97.042215. URL https://link.aps.org/doi/10.1103/PhysRevE.97.042215. Publisher: American Physical Society.

C. M. Leonard, H. M. Fuld, D. A. Frenz, S. A. Downie, J. Massague, and S. A. Newman. Role of transforming growth factor- in chondrogenic pattern formation in the embryonic limb: Stimulation of mesenchymal condensation and fibronectin gene expression by exogenenous TGF- and evidence for endogenous TGF--like activity. Developmental Biology, 145(1):99-109, May 1991. ISSN 0012-1606. doi: 10.1016/0012-1606(91)90216-P. URL http://www.sciencedirect.com/science/article/pii/001216069190216P.

M. Mojtahedi, A. Skupin, J. Zhou, I. G. Castao, R. Y. Y. Leong-Quong, H. Chang, K. Trachana, A. Giuliani, and S. Huang. Cell Fate Decision as High-Dimensional Critical State Transition. PLOS Biology, 14(12):e2000640, Dec. 2016. ISSN 1545-7885. doi: 10.1371/journal.pbio.2000640. URL https://journals.plos.org/plosbiology/article?id=10.1371/journal.pbio.2000640. Publisher: Public Library of Science.

J. D. Murray. Mathematical biology. I, volume 17 of Interdisciplinary Applied Mathematics. Springer-Verlag, New York, third edition, 2002. ISBN 0-387-95223-3.

S. A. Newman. Cell differentiation: What have we learned in 50 years? Journal of Theoretical Biology, 485:110031, Jan. 2020. ISSN 0022-5193. doi: 10.1016/j.jtbi.2019.110031. URL http://www.sciencedirect.com/science/article/pii/S0022519319304011.

S. A. Newman, T. Glimm, and R. Bhat. The vertebrate limb: An evolving complex of self-organizing systems. Progress in Biophysics and Molecular Biology, 137: 12-24, Sept. 2018. ISSN 0079-6107. doi: 10.1016/j.pbiomolbio.2018.01.002. URL http://www.sciencedirect.com/science/article/pii/S0079610717302857.

M. Ross, G. Kaye, and W. Pawlina. Histology: A Text and Atlas with Cell and Molecular Biology. Lippincott Williams \& Wilkins, 2002. ISBN 9780781751247. URL https://books.google.com/books?id=wrhYcf512igC.

I. Salazar-Ciudad, J. Jernvall, and S. A. Newman. Mechanisms of pattern formation in development and evolution. Development, 130(10):2027-2037, May 2003. ISSN 0950-1991, 1477-9129. doi: 10.1242/dev.00425. URL https://dev.biologists.org/content/130/10/2027. Publisher: The Company of Biologists Ltd Section: Review. 
D. Srivastava. Making or breaking the heart: from lineage determination to morphogenesis. Cell, 126(6): 1037-1048, Sep 2006.

A. Steinacher, D. G. Bates, O. E. Akman, and O. S. Soyer. Nonlinear Dynamics in Gene Regulation Promote Robustness and Evolvability of Gene Expression Levels. PLoS ONE, 11(4):e0153295, 2016.

S. Strogatz. Nonlinear dynamics and chaos: with applications to physics, biology, chemistry, and engineering. Studies in nonlinearity. Addison-Wesley Publ., Reading, Mass, 1994. ISBN 978-0-201-54344-5.

N. Suzuki, C. Furusawa, and K. Kaneko. Oscillatory protein expression dynamics endows stem cells with robust differentiation potential. PLoS ONE, 6(11):e27232, 2011.

R. Thom. Structural stability and morphogenesis. W. A. Benjamin, Inc., Reading, Mass.-London-Amsterdam, 1976. An outline of a general theory of models, Translated from the French by D. H. Fowler, With a foreword by C. H. Waddington, Second printing.

J. J. Tyson and B. Novak. Models in biology: lessons from modeling regulation of the eukaryotic cell cycle. BMC Biol., 13:46, Jul 2015.

J. J. Tyson, K. Chen, and B. Novak. Network dynamics and cell physiology. Nature Reviews Molecular Cell Biology, 2(12):908-916, Dec. 2001. ISSN 1471-0080. doi: 10.1038/35103078. URL https://www.nature.com/articles/35103078/boxes/bx2. Number: 12 Publisher: Nature Publishing Group.

J. J. Tyson, K. C. Chen, and B. Novak. Sniffers, buzzers, toggles and blinkers: dynamics of regulatory and signaling pathways in the cell. Current Opinion in Cell Biology, 15(2):221-231, Apr. 2003. ISSN 09550674. doi: 10.1016/S0955-0674(03)00017-6. URL https://linkinghub.elsevier.com/retrieve/pii/S0955067403000176.

H. Xiao and R. Wu. Quantitative investigation of human cell surface N-glycoprotein dynamics Electronic supplementary information (ESI) available: Two supplementary figures, and eleven supplementary tables. See DOI: 10.1039/c6sc01814a Click here for additional data file. Click here for additional data file. Chemical Science, 8(1):268-277, Jan. 2017. ISSN 2041-6520. doi: 10.1039/c6sc01814a. URL https://www.ncbi.nlm.nih.gov/pmc/articles/PMC5458730/.

\section{Appendix A : Illustrative simplified version of the system (2.17)- $(2.19)$}

To further aid visualization of the three-dimensional system (2.17)-(2.19), illustrated in Figure 2, we can consider an even simpler two component system which nevertheless displays some of the same key behaviors as the threecomponent system. For this, note that for $\pi=1$,

$$
\frac{d}{d t}\left(\frac{c_{1}}{c_{8}}\right)=L_{8}(t)-L_{1}(t)\left(\frac{c_{1}}{c_{8}}\right)^{2}
$$

This is a nonautonomous equation for $u(t)=c_{1}(t) / c_{8}(t)$ which then satisfies $\frac{d u}{d t}<0$ for $u(t)>\sqrt{L_{8} / L_{1}}$ and $\frac{d u}{d t}>0$ for $u(t)<\sqrt{L_{8} / L_{1}}$. Motivated by this and the fact that $L_{8}(t) \rightarrow 1 / \sigma$ as $t \rightarrow \infty$, we set up an illustrative 


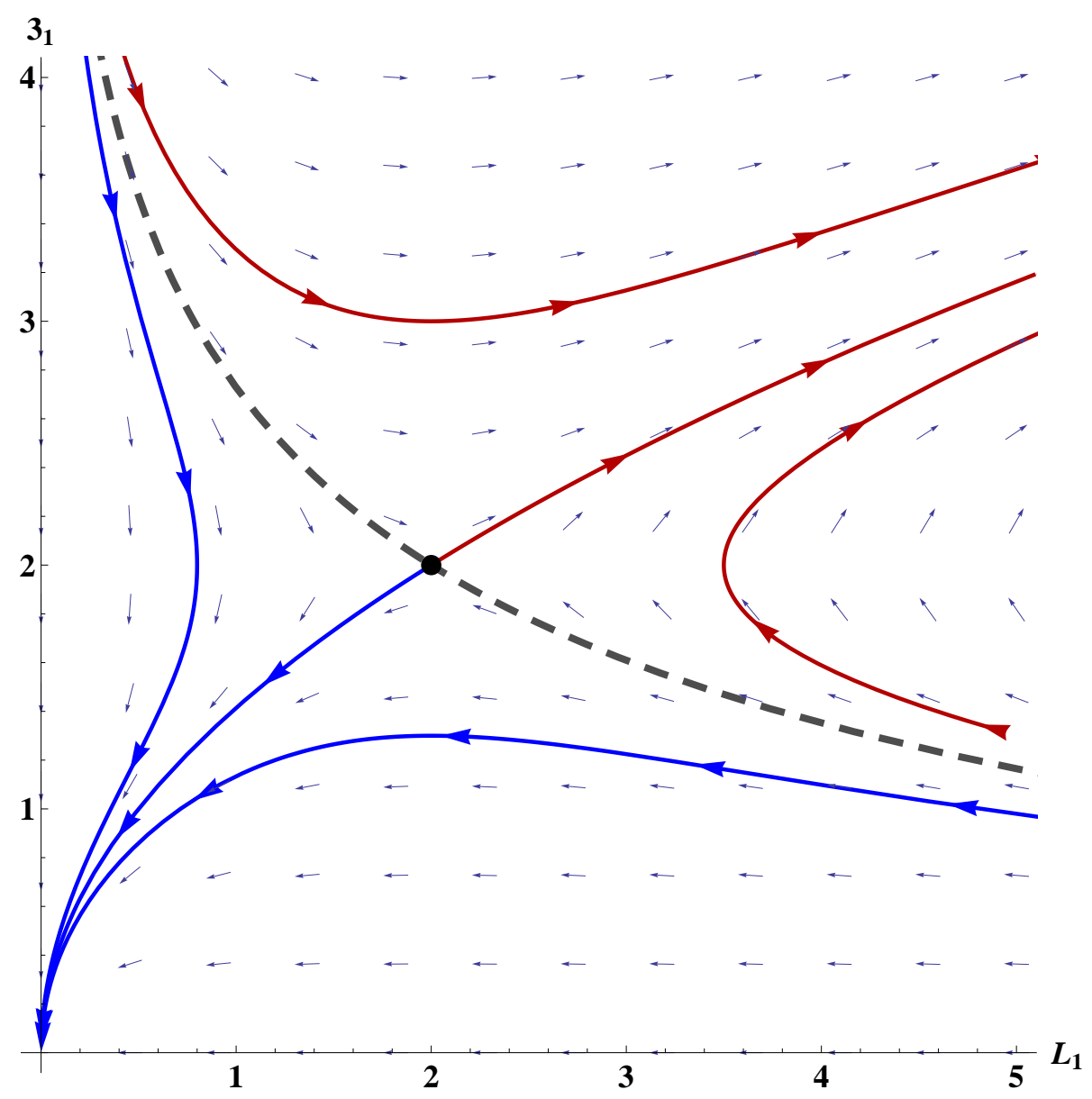

Figure 8: Phase plane for the toy model (Appendix A.1)-(Appendix A.2) for $\sigma=\rho=2$. The qualitative behavior is similar to the four dimensional system (2.13)-(2.16). Note the two regions corresponding to trajectories converging to $(0,0)$ and trajectories diverging to infinity in both component. The two regions are separated by the stable manifold of the saddle point (dashed).

toy model by taking the equations $(2.13)$ and $(2.15)$ for $L_{1}$ and $c_{1}$ and replace $c_{8}(t)$ by $\sqrt{\sigma L_{1}(t)} c_{1}(t)$. With this substitution, one obtains the two-component system

$$
\begin{aligned}
& \frac{d L_{1}}{d t}=\left(c_{1}-\rho\right) L_{1} \\
& \frac{d c_{1}}{d t}=\left(\sqrt{\frac{L_{1}}{\sigma}}-1\right) c_{1}
\end{aligned}
$$

A typical phase plane is shown in Figure 8. The phase plane shows some of the same key behaviors as the one for the four component system (2.13)-(2.16), namely the separation of phase space into two regions corresponding to different cell fates.

Note that the system (Appendix A.1)-(Appendix A.2) is very similar to simple Lotka-Volterra type population models of mutualism with negative internal growth rates, representing populations that depend on each other for mutual survival (Murray (2002)). 


\section{${ }_{565}$ Appendix B : Proof of Proposition 3.5}

We prove this result by matched asymptotic expansion. Let $r=\pi \rho$; so we are interested in the case $r \rightarrow \infty$.

By (3.2), the pair of complex eigenvalues $\lambda(\rho, \pi)$ and $\bar{\lambda}(\rho, \pi)$ are solutions of the polynomial equation

$$
0=r-(1+\pi) \lambda^{2}-\lambda^{3} .
$$

We seek an expansion in the form

$$
\lambda=\mu r^{\alpha}+\text { l.o.t. }
$$

where "l.o.t." denotes lower order terms in the power expansion in $r$. This results in the equation

$$
0=r-(1+\pi) \mu^{2} r^{2 \alpha}-\mu^{3} r^{3 \alpha} .
$$

Comparing powers of $r$, this forces $\alpha=1 / 3$, and thus $0=\mu^{3}-1$, which gives the three root $\mu_{1}=1, \mu_{2}=$ $\exp (2 \pi i / 3), \mu_{2}=\exp (4 \pi i / 3)$. We thus obtain the expansion of the complex eigenvalues as

$$
\lambda_{2 / 3}=-\frac{1}{2} \pm i \frac{\sqrt{3}}{2} r^{1 / 3}+\text { l.o.t. }
$$

from where the desired result follows:

$$
\lim _{\rho \rightarrow \infty}\left|\frac{\operatorname{Im}(\lambda)}{\operatorname{Re}(\lambda)}\right|=\sqrt{3}
$$




\section{${ }_{573}$ Supplementary material}

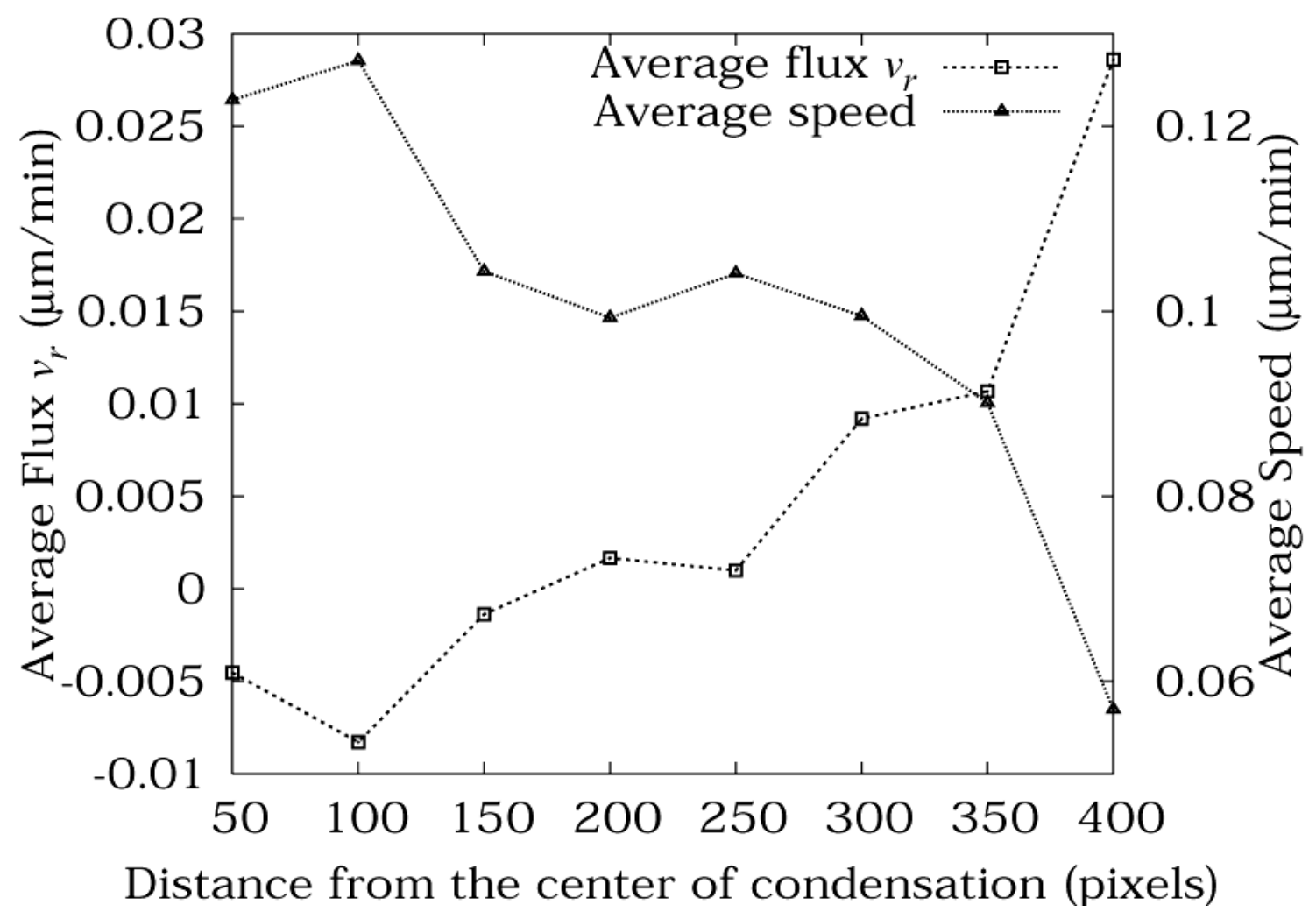

Figure S 1: Cell flux towards the center of condensation and average cell speed as a function of distance from the center of condensation. Cell flux in a band is defined as $v_{r}=\mathbf{v} \cdot \mathbf{r}$ averaged over all cells in the band over all 25 images in the image set, where $\mathbf{v}$ is the velocity of the cell and $\mathbf{r}$ is the unit vector pointing from the center of mass of the cell to the center of condensation.

Movie M1: Time-lapse movie of the emergence of protecondenstion taken at 2 min intervals for 480 minutes total. 